\title{
ART Network based Face Recognition with Gabor Filters
}

\author{
Md. Mozammel Haque \\ Dept. of Computer Science \& Engineering \\ Jahangirnagar University, \\ Savar, Dhaka - 1342, Bangladesh.
}

\author{
Md. Al-Amin Bhuiyan \\ Dept. of Computer Science \& Engineering \\ Jahangirnagar University \\ Savar, Dhaka - 1342, Bangladesh.
}

\begin{abstract}
Face recognition is emerging as an active research area in numerous commercial and law enforcement applications. Different existing methods perform well under certain conditions. Gabor-based face representation has achieved enormous success in face recognition. This research addresses a novel algorithm for face recognition using neural networks trained by Gabor features. The system is commenced on convolving a face image with a series of Gabor filter coefficients at different scales and orientations. The novel contribution of this paper is the scaling of rms contrast. The neural network employed for face recognition is based on the multilayer perceptron (MLP) architecture with ART algorithm. The effectiveness of the algorithm has been justified over a face database with images captured at different illumination conditions.
\end{abstract}

\section{Keywords}

Gabor filter, rms contrast, neural network, ART.

\section{INTRODUCTION}

Face representation using Gabor features has occupied an emerging research area in numerous commercial and law enforcement applications. The principal motivation to use Gabor filters is biological relevance that the receptive field profiles of neurons in the primary visual cortex of mammals are oriented and have characteristic spatial frequencies. Gabor filters can exploit salient visual properties such as spatial localization, orientation selectivity, and spatial frequency characteristics [1][2]. Considering these overwhelming capacities and its great success in face recognition, this paper addresses Gabor features to represent the face image and produces recognition task in tandem with neural network.

A fair amount of research works have been published in literature for Gabor based image recognition. Lades et al. developed a Gabor wavelet based face recognition system using dynamic link architecture (DLA) framework which recognizes faces by extracting Gabor jets at each node of a rectangular grid over the face image [3]. In the EBGM algorithm, the face is represented as a graph, each node of which contains a group of coefficients, known as jets. However, both LDA and EBGM require extensive amounts of computational cost. Liu and Wechsler have developed a Gabor feature based classification protocol using the Fisher linear discriminant model for dimension reduction [5]. Shan et al. have developed an enhanced fisher model using the AdaBoost strategy for face recognition [6]. Zhang et al. proposed a face recognition method using histogram of Gabor phase pattern [7]. Al-Amin et al. developed a multi-layer perceptron with backpropagation algorithm based neural network based Gobor filter method for face recognition [8].

This paper proposes a Gabor filter coefficient based ART neural network approach for face recognition. Since rms contrast is sensible for image representation, attempts are focused on rms scaling. The scaling of rms contrast produces better recognition performance. Despite robustness, Gabor filter based feature selection methods are normally computationally expensive due to high dimensional Gabor features. That's why the binary versions of the Gabor images performed by patterning has been employed as the input layer of neural network. The rest of the paper is organized as follows. Section 2 describes the overall system architecture and Section 3 image pre-processing. Section 4 describes Gabor filter design. Section 5 describes the patterning technique. Section 6 illustrates the construction ART neural network algorithm. The experimental method and results are presented in Section 7. Finally, the results are discussed, conclusions are drawn and future works are proposed in section 8.

\section{SYSTEM ARCHITECTURE}

The system proposed in this research is designed for recognition of face images. The system consists of five modules: a) Face extraction b) Face preprocessing c) Facial feature extraction using Gabor filter d) Binarization using patterning e) Face recognition using adaptive resonance theory algorithm (ART). The overall system architecture is shown in Fig. 1. 


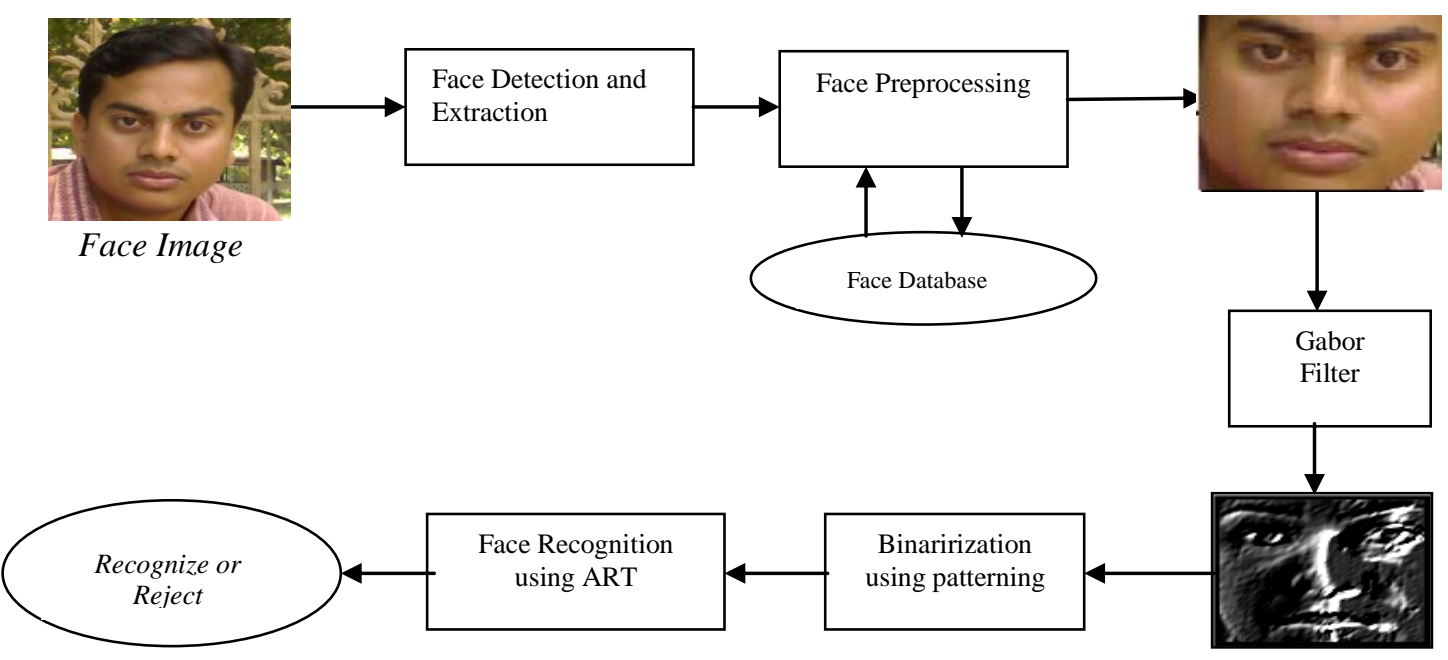

Fig. 1 A framework for face recognition.

Gabor Filtered

\section{IMAGE PRE-PROCESSING}

The original images are first converted into gray scale images. Pointing the centers of two eyes on each face image, all images are properly rotated, translated, scaled and cropped into $100 \times 100$ pixels. Images are then subjected to some image pre-processing operations. The image pre-processing phase includes contrast and illumination equalization, histogram equalization, and fuzzy filtering.

\subsection{Contrast and Illumination Equalization}

Contrast is a measure of the human visual system sensitivity. To achieve an efficient and psychologically-meaningful representation, all images are processed with same illumination and rms contrast.The rms (root mean square) contrast metric, equivalent to the standard deviation of luminance, is given by [8]:

$$
C_{r m s}=\left[\frac{1}{n} \sum_{i=1}^{n}\left(x_{i}-\bar{x}\right)^{2}\right]^{1 / 2}
$$

Where $x_{i}$ is a normalized gray-level value such that $0<x_{i}<1$ and $\bar{x}$ is the mean normalized gray level. With this definition, images of different human faces have the same contrast if their rms contrast is equal. The rms contrast does not depend on spatial frequency contrast of the image or the spatial distribution of contrast in the image. All images are maintained with the same illumination and same rms contrast using the following equation:

$$
\mathbf{g}=\alpha \mathbf{f}+\beta
$$

where $\alpha$ is the contrast and $\beta$ is the brightness to be increased or decreased from the original image $f$ to the new image $g$. The values of $\alpha$ and $\beta$ are chosen empirically. The illumination and rms contrast equalization process is illustrated in Fig. 2.

\subsection{Histogram Equalization}

The face images may be of poor contrast because of the limitations of the lighting conditions. So histogram equalization is used to compensate for the lighting conditions and to improve the contrast of the image [9].
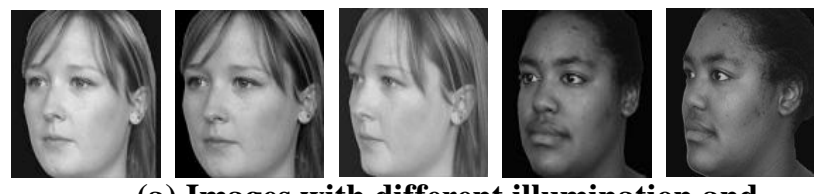

(a) Images with different illumination and
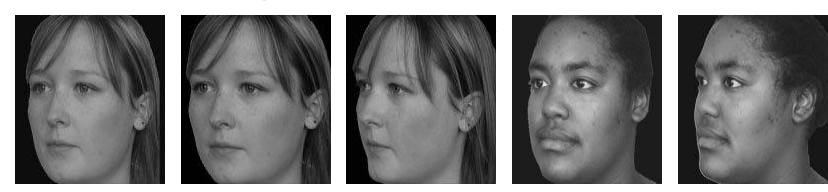

(b) Images with same illumination and same rms contrast.

Illumination and rms contrast equalization. Images were captured at illumination angles of $-38.4^{\circ},-21.6^{\circ},-0.2^{\circ}, 20.6^{\circ}, 37^{\circ}$, respectively, all images at a pose of $0^{\circ}$ (Courtesy: University of Hull, UK).

\section{DESIGNING GABOR FILTER}

Gabor filter works as a bandpass filter for the local spatial frequency distribution, achieving an optimal resolution in both spatial and frequency domains. The 2D Gabor filter $\psi_{f, \theta}(x, y)$ can be represented as a complex sinusoidal signal modulated by a Gaussian kernel function as follows [11]:

$\psi_{f, \theta}(x, y)=\exp \left[-\frac{1}{2}\left\{\frac{x_{\theta_{n}}^{2}}{\sigma_{x}^{2}}+\frac{y_{\theta_{n}}^{2}}{\sigma_{y}^{2}}\right\}\right] \exp \left(2 \pi f x_{\theta_{n}}\right)$,

where, $\left[\begin{array}{l}x_{\theta_{n}} \\ y_{\theta_{n}}\end{array}\right]=\left[\begin{array}{cc}\sin \theta_{n} & \cos \theta_{n} \\ -\cos \theta_{n} & \sin \theta_{n}\end{array}\right] \cdot\left[\begin{array}{l}x \\ y\end{array}\right]$

$\sigma_{x}, \sigma_{y}$ are the standard deviations of the Gaussian envelope along the $\mathrm{x}$ - and $\mathrm{y}$-dimensions, $\mathrm{f}$ is the central frequency of the sinusoidal plane wave, and $\theta_{n}$ the orientation. The rotation of 
the x-y plane by an angle $\theta_{n}$ will result in a Gabor filter at the orientation $\theta_{n}$. The angle $\theta_{n}$ is defined by:

$$
\theta_{n}=\frac{\pi}{p}(n-1)
$$

for $\mathrm{n}=1,2, \ldots, \mathrm{p}$ and $p \in \in_{\mathrm{N}}$, where $\mathrm{p}$ denotes the number of orientations.

Design of Gabor filters is accomplished by tuning the filter with a specific band of spatial frequency and orientation by appropriately selecting the filter parameters; the spread of the

filter $\sigma_{x}, \sigma_{y}$, radial frequency $\mathrm{f}$, and the orientation of the filter $\theta \mathrm{n}$. The Gabor representation of a face image is computed by convolving face image with the Gabor filters [12]. Let $f(x, y)$ be the intensity at the coordinate $(\mathrm{x}, \mathrm{y})$ in a gray scale face image, its convolution with a Gabor filter $\psi_{f, \theta}(x, y)$ is defined as:

$$
g_{f, \theta}(x, y)=f(x, y) \otimes \psi_{f, \theta}(x, y)
$$

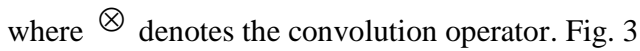
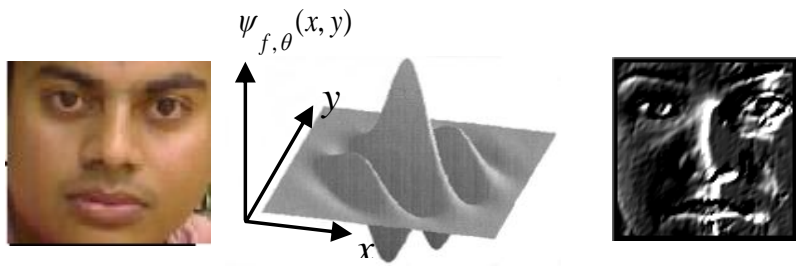

Fig. 3

illustrates the convolution result of a face image with a Gabor filter. The response to each Gabor kernel filter representation is a complex function with a real part $\mathfrak{R}\left\{g_{f, \theta}(x, y)\right\}$ and an $\begin{array}{ll}\text { imaginary part } & \mathfrak{J}\left\{g_{f, \theta}(x, y)\right\} \text {. The magnitude response }\end{array}$ $\left\|g_{f, \theta}(x, y)\right\|$ is expressed as:

$\left\|g_{f, \theta}(x, y)\right\|=\sqrt{\mathfrak{R}^{2}\left\{g_{f, \theta}(x, y)\right\}+\mathfrak{I}^{2}\left\{g_{f, \theta}(x, y)\right\}}$

This research uses the magnitude response $\left\|g_{f, \theta}(x, y)\right\|$ to represent the features. To reduce the influence of the lighting conditions, the output of Gabor filter about each direction has been normalized. In the sequel, a transformation $Q_{f, \theta}(x, y)$ to which $\left\|g_{f, \theta}(x, y)\right\|$ is subjected is given by: Convolution result of a face image with a Gabor filter. (a) Face image, (b) Gabor filter ( $\mathrm{f}=0.19, \theta=3 \pi / 4, \sigma \mathrm{x}=\sigma \mathrm{y}=3$ ), (c) Output of Gabor filter.

$$
Q_{f, \theta}(x, y)=\frac{\| g_{f, \theta^{(x, y)} \|}}{\sum_{\theta \in \Theta}\left\|g_{f, \theta}(x, y)\right\|} .
$$

The important issue in the design of Gabor filters for face recognition is the choice of filter parameters. This research organizes 15 Gabor channels consisting of five orientation

parameters

$$
\theta \in\left\{0, \frac{\pi}{5}, \frac{2 \pi}{5}, \frac{3 \pi}{5}, \frac{4 \pi}{5}\right\}
$$

frequencies $f \in\{0.06,1.0,1.4\}$, respectively. and three spatial

\section{BINIRIZATION USING PATTERNING}

Patterning involves replacing each pixel by a pattern taken from a 'binary font'. Consider the following $3 \times 3$ font pattern, as shown in Fig. 4.

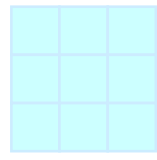

0

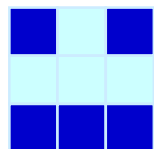

5

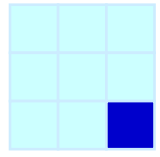

1

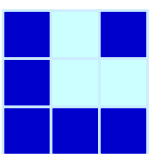

6

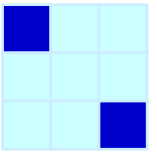

2

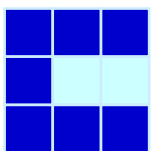

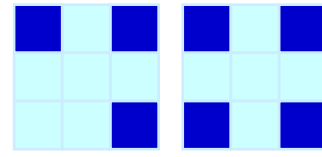

3

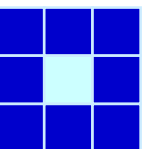

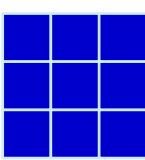

Fig. 4 Binary fonts for generating patterns

These fonts are used to print an image consisting of ten gray levels. Since we are using replacing each pixel by a $3 \times 3$ block of pixels, both the width and the height of the image is increased by a factor of 3 . The algorithm for the patterning process is given below and the output of a typical face image is shown in Fig. 5.

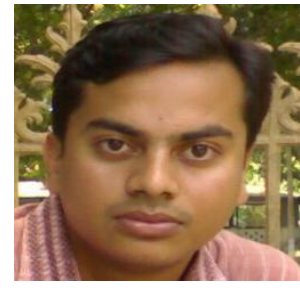

(a) Original image

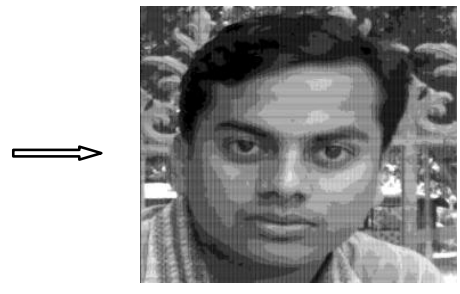

(b) Patterned image
Fig. 5 Patterning process.

\section{FACE RECOGNITION USING ART}

ART encompasses a wide variety of neural networks. The basic ART System is an unsupervised learning model. ART matching leads to this resonant state, which permits learning. The network learns only in its resonant state. ART neural networks are capable of developing stable clusters of arbitrary sequences of input patterns by self-organizing. The ART1 simplified model consists of two layers of binary neurons (with values 1 and 0 ), called F1 (the comparison layer) and F2 (the recognition layer). 
Each neuron in F1 is connected to all neurons in F2 via the continuous-valued forward long term memory (LTM) Wf, and vice versa via the binary-valued backward LTM $\mathrm{Wb}$. The other modules are gain 1 and 2 (G1 and G2), and a reset module. Each neuron in the comparison layer receives three inputs.

\section{EXPERIMENTAL RESULTS AND PERFORMANCE}

In order to evaluate the effectiveness of the proposed method, experiments were carried out for real images at different illumination conditions. We used CMU Pose, Illumination, and Expression (PIE) database [13] and selected 200 images of 40 individual subjects with two different poses and five different illumination conditions.

Half of the images in the database were used as a training dataset and the remaining images were used as probe images in the recognition test. All images were subjected to Gabor filters and were convolved with 15 Gabor filters. To each face image, the outputs were 15 images which record the magnitudes of the Gabor filter responses. Fig. 7 shows the results for a typical face image.

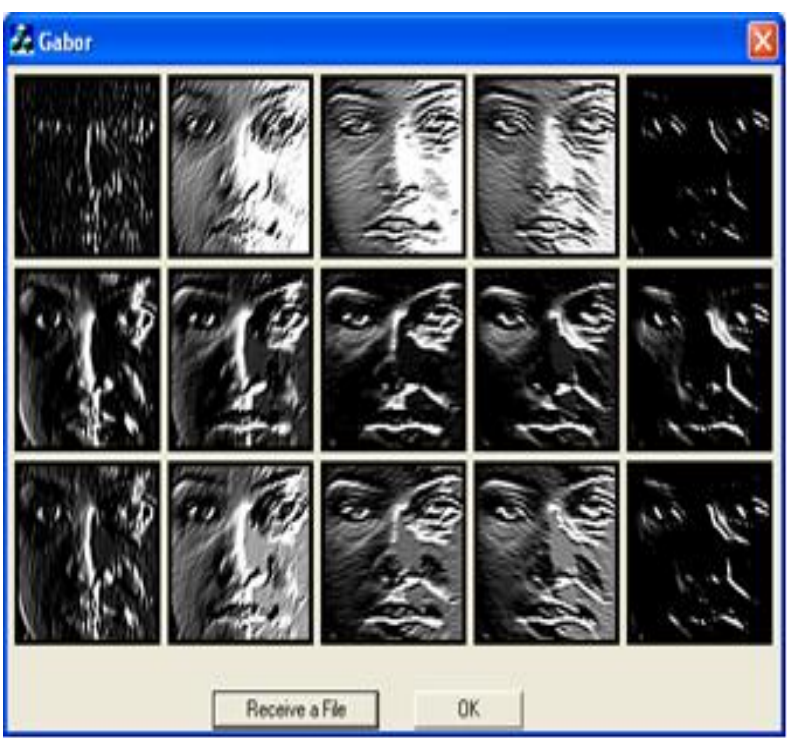

Fig. 7 Gabor filter response of a typical face image.

The output of the Gabor filters were used to train the neural network. The training curve, indicating the gradual reduction in error over several epochs due to ART learning algorithm, is shown in Fig. 8. Comparing the performance of two networks BPNN, BPNN with BAM and ART algorithm reveals that the proposed ART network takes less iteration than BPNN and hybrid network in completion of the training process.

Finally, the effect of the proposed algorithm on the performance of face recognition has been analyzed. In order to evaluate the method for contrast equalization, we performed a comparison among our proposed system (ART, rms scaling Gabor), the Elastic Bunch Graph Matching (EBGM), and the log-polar Gabor methods. The comparison results for the average recognition rates using different methods are furnished in Table I.

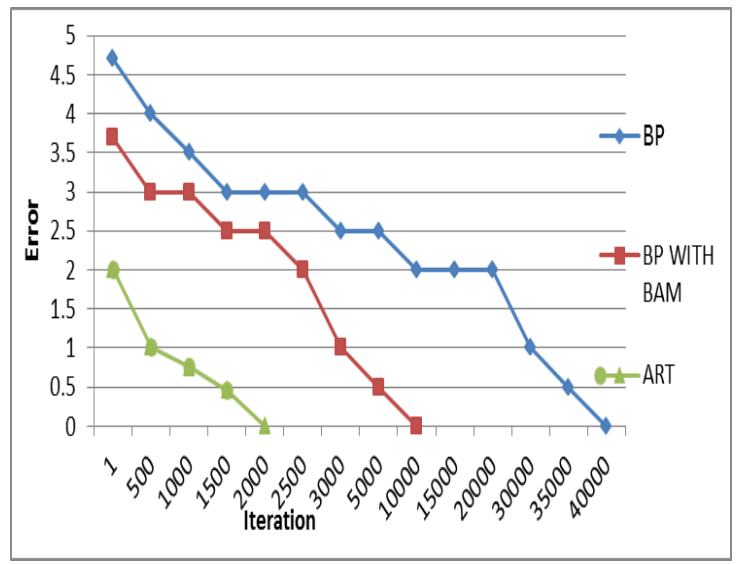

Fig 8: Error versus iteration

Table I Recognition Performance Of The Proposed Algorithm

\begin{tabular}{lll}
\hline \hline METHOD & $\begin{array}{l}\text { CORRECT } \\
\text { RECOGNITION } \\
(\%)\end{array}$ & $\begin{array}{l}\text { Correct } \\
\text { Rejection } \\
(\%)\end{array}$ \\
\hline EBGM & 75.29 & 78.32 \\
Log-polar & 77.38 & 82.33 \\
Neural net based & 81.50 & 82.75 \\
Gabor & & 85.50 \\
Hybrid net based & 84.50 & \\
Gabor & & 93.2 \\
ART Gabor & 91.2 & \\
\hline \hline
\end{tabular}

\section{CONCLUSION}

This paper presents a neural network based face recognition system using Gabor filter coefficients that can cope with illumination changes. The recognition performance has been improved substantially due to implication of contrast equalization using the rms value of the image pixels. ART Application rather than hybrid network (BAM and BPNN) and BPNN takes less iteration to train and less time to recognize faces. Since each pixel of the magnitude response of Gabor filter corresponds to a Gabor feature, the number of Gabor features for each sample is $100 \times 100 \times 15=150,000$. Therefore, our next step will be to improve the algorithm which would be able to employ more complex classifiers and distance measures to represent Gabor faces with spatial and frequency features.

\section{REFERENCES}

[1] J.G. Daugman, "Uncertainty Relation for Resolution in Space, Spatial Frequency, and Orientation Optimized by Two-Dimensional Visual Cortical Filters", Journal of Optical Society America A, Vol. 2, No. 7, 1985, pp. 1160 1169.

[2] J. Buhmann, J. Lange, and C.V. Malsburg, "Distortion invariant object recognition by matching hierarchically labeled graphs", Procedings of International Conference on Neural Neural Networks, Washington DC, 1989, pp. 155159.

[3] M. Lades, J.C. Vorbruggen, J. Buhmann , J. Lange, C.V. Malsburg, C. Wurtz and W. Konen, "Distortion invariant object recognition in the dynamic link architecture", IEEE Trans. Computers, Vol. 42, No. 3, 1993, pp. 300-311.

[4] L. Wiskott, J.M. Fellous, N. Kruger, and C.V. Malsburg, "Face recognition by elastic bunch graph matching", IEEE 
Trans. Pattern Anal. Mach. Intell., Vol. 19, No. 7, 1997, pp. 775-779.

[5] C. Liu, and K. Wechsler, "Gabor feature based classification using the enhanced Fisher linear discriminant model for face recognition", IEEE Trans. Image Process., Vol. 11, No. 4, 2002, pp. 467-476.

[6] S. Shan, P. Yang, X. Chen and W. Gao, "AdaBoost Gabor Fisher classifier for face recognition", Proc. IEEE Int. Workshop Analysis and Modeling of Faces and Gestures, 2005, pp. 278-291.

[7] B. Zhang, S. Shan, X. Chen, W. Gao, "Histogram of Gabor phase patterns: a novel object representation approach for face recognition", IEEE Trans. Image Process., Vol. 11, No. 4, 2002, pp. 467-476.

[8] Md. Al-Amin Bhuiyan and Chang Hong Liu, "On Face Recognition using Gabor Filters", Proceedings of World Academy of Science, Engineering and Technology, Vol. 22, July, pp. 51-56, 2007.
[9] E. Peli, "Contrast in Complex Images", Journal of Optical Society, Vol. 7, No. 10, 1990, pp. 2032-2040.

[10] J. Jantzen, "Tutorial on Fuzzy Logic", www.iau.dtu.dk/ jj/pubs/logic.pdf.

[11] T. Andrysiak, and M. Choras, "Image retrieval based on hierarchical Gabor filters", International Journal of Mathematics and Computer Science, Vol. 15, No. 4, 2005, pp. $471-480$

[12] M. Zhou, and H. Wei, "Face verification using Gabor Wavelets and AdaBoost", $18^{\text {th }}$ International conference on Pattern Recognition, 2006, pp. 404-407.

[13] T. Sim, S. Baker and M. Bsat, "The CMU Pose Illumination and Expression (PIE) Database", Proceedings of the 5th International Conference on Automatic Face and Gesture Recognition, 2002. 